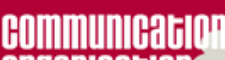

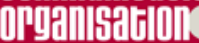

\section{Communication et organisation}

Revue scientifique francophone en Communication

organisationnelle

$53 \mid 2018$

Digitalisation et recrutement : perspectives

informationnelles et communicationnelles

\title{
Identité et ethos discursif du candidat dans l'espace numérique
}

Identity and discursive ethos of candidates in the digital space

\section{Elena Sekhniachvili-Komperdra et Ludovic Chatenet}

\section{(2) OpenEdition \\ 12 Journals}

Édition électronique

URL : https://journals.openedition.org/communicationorganisation/6553

DOI : 10.4000/communicationorganisation.6553

ISSN : $1775-3546$

Éditeur

Presses universitaires de Bordeaux

\section{Édition imprimée}

Date de publication : 1 juin 2018

Pagination : 71-89

ISBN : 979-10-300-0302-4

ISSN : 1168-5549

Référence électronique

Elena Sekhniachvili-Komperdra et Ludovic Chatenet, « Identité et ethos discursif du candidat dans l'espace numérique », Communication et organisation [En ligne], 53 | 2018, mis en ligne le 01 juin 2021, consulté le 28 janvier 2022. URL : http://journals.openedition.org/communicationorganisation/6553 ; DOI : https://doi.org/10.4000/communicationorganisation.6553 
DOSSIER

\title{
Identité et ethos discursif du candidat dans l'espace numérique
}

\author{
Elena Sekhniachvili-Komperdra ${ }^{1}$, Ludovic Chatenet ${ }^{2}$
}

Résumé : La modification des pratiques de recrutement liée à l'utilisation des technologies numériques et de l'internet pose un problème de mise en place des stratégies de visibilité et de valorisation des candidats à l'emploi. L'analyse de leurs discours numériques sur différentes plateformes permettra : (1) de décrire les conditions de l'émergence de leur identité qui s'ajuste aux pratiques et aux supports ; (2) de définir leurs styles stratégiques (identité idem/ipse) qui donne accès à leurs systèmes de valeurs. Les styles dégagés montrent comment la construction d'un profil sur internet renseigne sur l'éthique du candidat.

Mots-clés : recrutement, identité idem/ipse, discours numérique, sémiotique, accommodations stratégiques.

Identity and discursive ethos of candidates in the digital space

Abstract: The modification of recruitment practices due to the use of digital technologies and the Internet raises the problem of strategies of the applicants' exposure and bighlighting. The analysis of their digital discourse through different platforms will allow us 1) to describe the conditions of this identity emergence adjusted to practices and electronic support; 2) to define their strategic styles (idem/ipse) that give access to detecting the applicants'sets of values. These styles display how the construction of profiles on the Internet leads to better understanding the candidates' ethics.

Keywords: recruitement, identity idem/ipse, digital discourse, semiotics, strategic accomodations.

1- Elena Sekhniachvili-Komperdra est docteure en sciences du langage, spécialité sémiotique. Elle est aujourd'hui chercheuse associée au laboratoire CeReS de l'Université de Limoges. Ses recherches sont consacrées à l'analyse du discours promotionnel (publicité, packaging, discours numérique) et sa dimension éthique.

2- Ludovic Chatenet est docteur en sciences du langage, spécialité sémiotique. Il est aujourd'hui membre associé du laboratoire MICA de l'Université Bordeaux Montaigne. Ses travaux portent sur la dynamique de l'interculturalité du point de vue de la sémiotique visuelle et de la sémiotique de la culture. 


\section{Introduction}

À l’ère des « nouvelles technologies ", l'identité numérique est l'enjeu majeur de la modification des pratiques de recrutement et l'apparition de nouvelles techniques de repérage et de gestion de l'identité sur les réseaux. Les candidats, autant que les entreprises, doivent être attentifs à leur notoriété en ligne (e-réputation). Ainsi, le processus de recrutement implique des stratégies de présentation de soi relatif à la déclinaison de l'identité numérique sur différents supports et plateformes. La réussite du candidat dépendra de sa capacité à assurer la cohérence de son identité, présentant son parcours, ses valeurs et ses projets, mais également d'assurer sa conformité avec les attentes et valeurs de l'entreprise. Questionner la construction de la réputation du candidat implique d'étudier l'expression des valeurs dans ses discours mais aussi dans ceux de l'entreprise. Elle amène également à considérer que l'approche identitaire et l'approche éthique sont inséparables.

De nombreux travaux ont été consacrés aux problèmes de la construction et de la régulation de l'identité numérique en essayant de «mettre en lumière les ressorts et dilemmes de la présence en ligne et de l' "être ensemble" » (Fourmentraux 2015). Cependant la question de l'identité comme production discursive saisie dans sa dimension narrative -définie par sa cohérence, sa cohésion et sa congruence interne- n'a pas encore fait l'objet de recherches dans le domaine de la communication propre au recrutement.

Dans cet article, nous étudierons les conditions et les formes de la présentation de soi des candidats dans l'espace numérique. Nous porterons une attention particulière aux contraintes qui imposent de nombreux ajustements à cette identité dans la mesure où celle-ci s'intègre dans différentes pratiques et supports numériques. Notre analyse s'intéressera d'une part aux conditions d'émergence de l'identité dans le discours numérique et d'autre part, à la façon dont celle-ci manifeste une forme de vie. Pour cela, nous décrirons la situation du recrutement, la mise en forme des identités et des récits de vie des candidats sur une plateforme numérique comme une pratique d'accommodation avec l'identité des entreprises. De manière générale, nous défendons l'idée que le récit numérique des candidats donne l'accès à leur forme de vie, leur style identitaire qui définit leur façon d'être, d'agir et d'interagir avec l'autre. Une fois ce style défini il est possible d'évaluer sa compatibilité avec les attentes et les valeurs de l'entreprise. 
Pour mener cette analyse nous avons constitué un corpus de quelques sites internet destinés au recrutement, des plateformes spécialisées dans l'emploi (Pôle Emploi, Monster), des réseaux professionnels (LinkedIn) et des sites d'annonce en ligne (Leboncoin). L'étude plus spécifique des styles expérientiels des candidats a été effectuée à partir d'un échantillon de 100 profils disponibles sur LinkedIn et Pôle-emploi.

\section{Approche méthodologique}

Entendu que l'espace numérique est non seulement un medium véhiculant les discours des candidats et des employeurs (coénonciateurs) mais aussi un espace stratégique de communication, nos travaux mobiliseront le modèle sémiotique qui étudie la signification en considérant que les langages et les pratiques signifiantes sont des pratiques sociales (Floch 1990 : 4). Dans ce cadre, nous considérons qu'une énonciation numérique reflète une situation de communication saisissable à partir des traces (marques) linguistiques dans le discours ${ }^{3}$. Nous rejoignons alors certains chercheurs en sciences de l'information et de la communication qui considèrent que les traces donnent accès à l'étude de l'expérience de l'homme (Galinon-Mélenec, Monseigne 2011, Jeanneret 2011 : 70). Nous considérons donc que les traces laissées sur internet par un candidat-usager mettent en récit son parcours et son projet de vie. Lénonciation devient porteuse de «formes identitaires » en faisant référence aux traces de subjectivité dans le langage (Benveniste 1974, Kerbrat-Orrecchioni 1980). Cela signifie que la présence du locuteur (le candidat) se manifeste dans le discours numérique à partir de sa prise de position qui s'affiche au nom du « je » et organise également l'espace social des relations entre « je », « tu » et « il » (Benveniste 1974 : 67, Beyaert-Geslin 2013). Dans notre démarche sémiotique, nous allons plus particulièrement utiliser le modèle de Fontanille (Fontanille 2011) qui permet d'étudier les identités à partir des traces.

3- L'approche énonciative en sémiotique mobilise en premier lieu la théorie de Benveniste (1966) qui conçoit l'énonciation, c'est à dire l'acte de production de l'énoncé, par le biais de marques linguistiques de la personne, de l'espace et du temps (je, ici, maintenant/ on, ailleurs, alors). Les recherches récentes sur les pratiques considèrent ces marques pour déterminer le positionnement d'instances énonciatives et donc de l'émergence de leur identité en discours. (Fontanille 2008). 


\section{L'identité discursive et l'identité numérique}

L'analyse de l'inscription de l'identité sur le support digital doit s'intéresser à la manière dont celle-ci exprime des valeurs, s'ajuste et se transforme; autrement dit, comment elle construit et manifeste des styles stratégiques. Pour décrire le style identitaire du candidat à travers le récit qu'il construit en ligne, nous nous appuierons sur le modèle d'analyse de l'identité dans le discours proposé par Fontanille (Fontanille 2011) qui permet de décrire cette identité comme une entité éthique. À l'origine, Ricœur distingue deux composantes de l'identité personnelle qui sont irréductibles l'une à l'autre : (1) l'identité comme mêmeté (latin : idem, the same), c'est-à-dire un caractère propre compris comme un ensemble de traits distinctifs permettant de réidentifier un individu comme étant le même ; (2) l'identité comme ipséité (latin : ipse) qui implique un sentiment de continuité du " je ", c'est-à-dire une fidélité à Soi à travers le temps (Ricœur 1990). En intégrant ces principes, le modèle de Fontanille nous permet de distinguer trois types d'identité (Fontanille $2006: 85-86)$ :

1) le Moi-chair : « instance siège de la sensori motricité », centre de référence de la perception du sensible.

2) le Soi-idem correspond à « l'identité des rôles, dans la perspective de la saisie », qui implique l'espace et le temps. Il s'agit d'une identité fondée sur « un principe de répétition et de similitude».

3) le Soi-ipse correspond à « l'identité des attitudes, dans la perspective de la visée ». Elle se fonde sur l'accumulation progressive de traits transitoires dans la mesure où le sujet dans l'acte, et aux prises avec l'éthique narrative, se découvre « autre».

Cette distinction peut permettre d'observer l'identité « en devenir » du candidat en mesurant la cohésion, la cohérence et la congruence ${ }^{4}$ de son action en ligne. Dans le modèle utilisé, la cohésion de l'action repose sur la confrontation entre d'un côté, les différentes phases du Moi-chair (recherche de valeurs, quête axiologique) et de l'autre, le principe de répétition-similitude du Soi-idem, c'est-à-dire une programmation narrative (rôles définis). La cohérence de l'action repose sur le guidage des phases du Moi-chair par le principe de visée permanente qui

4- Pour rappel, la cohésion est un effet local qui renvoie la relation entre les parties; la cohérence est un effet global d'unité et de continuité; la congruence renvoie au rapport des parties au tout. 
caractérise le Soi-ipse (confrontation avec les autres rôles). La congruence de l'action repose quant à elle sur la confrontation entre les deux modes de construction du Soi (la répétition des rôles similaires d'un côté, et la permanence de la visée de l'autre) (Fontanille 2011 : 26). Par exemple, le candidat qui a exercé le même métier toute sa vie assume le même rôle et manifeste la stabilité et la constance de l'identité idem au niveau du caractère. Cependant le candidat peut changer de rôles (les métiers) mais garder toujours le même objectif (la visée) qui définit son projet de vie ${ }^{5}$. Ces paramètres font alors émerger des styles stratégiques observables.

\section{La présence du candidat et de l'employeur sur le Net}

La présence des traces des instances discursives et leur interaction se manifestent dans le discours numérique à travers différents niveaux de pertinences $^{6}$ (Fontanille 2008) qui en constituent des niveaux d'analyse. Ces derniers définissent les contraintes qui préfigurent la mise en forme d'une identité en ligne et orientent la pratique d'interprétation de l'énoncé.

Tout d'abord, l'identité peut apparaître comme un signe (unité de sens minimale) ; par exemple, le nom propre, l'avatar ou d'autres signes identitaires (verbaux et iconiques) renvoyant à l'instance qui parle (recruteurs ou candidats). L’employeur se présente généralement par un nom, parfois un logo (identité institutionnelle), souvent complété par le lieu géographique de l'entreprise. Les désignations des énonciateurs sont diverses : «Gery, Ben, MARIE 871 », une photo et le patronyme (par opposition au pseudonyme), comme on peut le voir sur le site LinkedIn. Les candidats peuvent être identifiés par leur métier (secrétaire) ou une action (protection des animaux). Ils peuvent laisser une trace de leur jugement en forme d'icône (like/dislike).

En poursuivant avec le modèle de Fontanille, on doit considérer qu'au moment où les signes s'organisent en syntaxe, ils constituent des textes. La particularité du texte numérique réside dans son épaisseur discursive relative à sa nature hypertextuelle.

Les employeurs et les candidats s'expriment par des textes qui sont réunis soit au sein de la même plateforme d'embauches : les job-boards (Pôle emploi,APEC, Monster, etc.), soit dispersés sur les réseaux. L'espace

5- L'exemple éloquent est fourni par Floch « Des jumeaux si différents, si semblables. L'identité selon Waterman " (Floch 1995).

6- Les niveaux de pertinence distingués par Fontanille sont : les signes, les textes-énoncés, les objets, les scènes pratiques, les stratégies et les formes de vie (Fontanille 2008 : 18-36). 
numérique a la particularité de s'appuyer sur un moteur de recherche qui trie l'information selon une logique propre (algorithme) permettant de réunir les informations au moyen de mots-clés, qui sont des signes.

L'espace numérique n'est pas seulement une surface d'inscription des textes, c'est aussi un espace d'action car les usagers agissent via l'Internet. Il implique donc deux procès dont il faut rendre compte : (1) l'interprétation du texte inscrit (le contenu d'une page) et (2) la manipulation de l'objet (cliquer sur les boutons, taper du texte) qui détermine la relation énonciative entre les partenaires de l'échange lors du processus du recrutement (rédiger et poster une offre, rechercher une offre, répondre, etc.). Lidentité du candidat apparaît ici à un autre niveau de pertinence - le niveau des pratiques - comme une identité agissante. Le candidat est engagé dans une pratique argumentative, une pratique collaborative, une pratique de recherches d'information voire d'autres types de pratiques connexes (payer et acheter en ligne, se déplacer, visiter les sites, etc.) qui, sans rapport direct avec le recrutement, laissent quand même des traces dans la mémoire collective digitale (mémoire de l'activité Web). Lidentité du candidat se manifeste comme un agent dans plusieurs pratiques et son parcours, composé de plusieurs actes, permet d'évaluer son profil sans que le candidat parle de lui. Ce parcours retracé par l'employeur devient un argument pour l'embaucher. Ainsi, le candidat perd le contrôle de sa pratique argumentative qui comprend désormais non seulement ses paroles mais aussi ses actes non contrôlés. Pour agir de façon efficace, le candidat doit alors ajuster son identité numérique en fonction des différentes contraintes imposées par les supports et les pratiques. Autrement dit, il doit procéder à des réglages stratégiques. Cette visée stratégique destinée à articuler des pratiques entre elles est ce que l'on nomme accommodation. La proposition de Fontanille distingue deux types d'accommodation : (1) l'autoaccomodation qui articule les actions du candidat avec son système personnel, son projet de vie par exemple) et (2) l'hétéroaccomodation qui tient compte du rapport entre les actions du candidat et le système axiologique extérieur (social), c'est-à-dire les normes, les règles tacites et implicites, ainsi que les règles déontologiques propres à chaque corps de métiers (Fontanille 2010). À ce niveau, on observe donc comment la pratique de présentation de soi sur les réseaux positionne le candidat dans une interaction sociale réglée.

Le niveau supérieur, que nous chercherons à atteindre dans cet article est celui des formes de vie. Pour Fontanille, il s'agit du niveau de la culture où l'identité apparaît comme une forme iconisée de comportement 
manifestant un style propre qui caractérise certaines valeurs (éthique). C'est à ce niveau, proprement social, que se confrontent les identités idem et ipse, et où la cohésion et la cohérence des choix réalisés émergent comme des formes reconnaissables.

\section{Les fluctuations identitaires (identité au niveau des pratiques)}

\section{Ajustements au support formel et au support matériel}

L'identité dans le discours est déterminée par la sélection des formes de l'expression et du contenu qui impliquent un ajustement avec le support et les pratiques. Nous allons donc étudier comment l'identité s'adapte au support. Nous partons de l'idée que l'Internet avec ses technologies digitales est un médium qui impose des contraintes à la production du sens, c'est-à-dire, lorsque les énoncés sont inscrits sur le support formel (le format de la page Web) et le support matériel (l'ordinateur, la tablette, le téléphone portable). Du point de vue de la sémiotique des pratiques, le support formel correspond au niveau du texte puisqu'il désigne un ensemble composite clôturé (avec un début et une fin) verbal et iconique. Dans notre cas, il s'agit du format de la page web décrit par son organisation tabulaire, topologique et dont certains éléments (typologie, formes d'encarts) permettent de définir le genre. Comme nous l'avons vu, les textes s'inscrivent sur des objets-supports, ou supports matériels, qui sont des corps dotés d'une matérialité, d'une morphologie mais aussi d'une fonctionnalité qui les ancre dans une pratique. De ce point de vue, les médias (internet) apparaissent comme des dispositifs matériels et sensibles que l'on peut manipuler (Pignier 2006, Dondero, Reyes-Garcia 2016) et dont les propriétés techniques permettent la lisibilité et l'interactivité des textes qu'ils portent. En définissant l'outil internet de cette manière, nous pouvons considérer que l'identité numérique est une identité fluctuante, autrement dit, « un ensemble de traits cherchant à se stabiliser sur un support d'inscription » (Dondero 2016).

Nous voudrions défendre l'idée que la mise en forme de l'identité des communicants et les effets de sens qu'elle produit sont prédéterminés par l'objet, le support sur lequel cette identité est apposée. Comme nous l'avons précisé déjà, les propriétés du support orientent l'énonciation du parcours du candidat. L'idée d'un cadre guide fournit par le medium (internet) et le type de site, nous invite à discuter les différents degrés de la liberté du candidat dans son choix du contenu et de la forme. Pour 
débuter notre enquête, nous allons d'abord comparer les présentations discursives et visuelles des candidats sur différentes plateformes :

\section{Exemple 1 : Leboncoin}

Notre premier cas est un candidat qui se définit par son pseudonyme : «Al» (anonymé et informel), par la date de la mise en ligne de son annonce " Mise en ligne le 23 janvier à $19: 36$ ", par le lieu « ville : Nedde 87120 » et par un titre : " cherche emploi divers », placé en haut de l'annonce, définissant son projet. Ce candidat n'a pas souhaité ajouter d'image. Voici le texte de l'annonce :

Homme de 26 ans titulaire du permis B C et CE avec Fimo.

expérience dans la mécanique 3 ans expérience dans l'agriculture fils

d'agriculteur.

cherche poste dans la conduite d'engins, camion ....

ou bien poste dans un service maintenance parc espace vert

je ne suis pas fermé au proposition j'étudie tout.

La première remarque que l'on peut formuler est l'absence relative de contrainte pour la mise en forme et le contenu de l'annonce : une zone de texte sans limite de caractères et une organisation simple (titre, corps de l'annonce, coordonnées). Le style de la présentation de soi est très libre.

Le texte donne une série d'informations sur l'annonceur : son identité est définie par son âge (26 ans) et par ses compétences : (1) ses savoirs (diplômes, formations) : "titulaire du permis B, C et CE avec Fimo » et (2) ses savoir-faire : "expérience dans la mécanique 3 ans, dans l'agriculture ». Sa compétence est également identifiée par le fait qu'il est fils d'agriculteur. L'argument désigne ici la filiation comme garantie de la transmission d'un savoir-faire et d'une connaissance profonde du milieu (héritage).

Le projet de vie du candidat n'est pas très clair : « cherche poste dans la conduite d'engins, camion... ou bien dans un service parc espace vert ». L'utilisation des « ... » suivie de « ou bien » manifeste une hésitation véhiculée par la syntaxe, mais aussi le lien entre deux thèmes (chauffeur, espace vert) présentés comme deux alternatives. La mise en forme se caractérise par une absence de structure, ce qui rend le propos un peu délié et difficile à suivre. 
Nous pouvons formuler quelques remarques sur l'ethos du candidat, c'est-à-dire sa manière d'être ou son attitude, telle quelle est manifestée par l'annonce qu'il a composée. L'ethos dont nous parlons ici émerge de l'énonciation et renvoie directement à ce que Maingueneau (2014) nomme ethos discursif. À l'instar de cet auteur, nous distinguerons deux types d'ethos discursif : (1) l'ethos dit : ce que le candidat dit sur lui-même, en terme de contenu, et (2) l'ethos montré : la manière dont le candidat communique son intentionnalité (mode de communication, choix des mots, des arguments, du registre). L'ethos dit ne permet pas ici de reconstruire son identité, en revanche, l'ethos montré est pertinent pour étudier cette annonce. Tout d'abord le style employé (syntaxe, registre) se rapproche du discours oral plutôt que de l'écrit, ce qui révèle que le candidat n'a pas beaucoup d'expérience dans la rédaction de textes. De plus, l'absence de majuscule, de ponctuation, la syntaxe incomplète (phrases inachevées) et l'orthographe caractérisent un texte peu scolaire permettant de déduire que le candidat a un faible niveau d'étude.

Que nous dit ce texte sur l'identité du candidat ? En rapportant ces dimensions de l'ethos aux paramètres de l'identité présentés dans notre méthodologie, on peut considérer que l'identité idem regroupe les traits d'identification de ce candidat : âge, diplômes, compétences. Son identité ipse a une tendance prononcée vers le changement et l'absence de projet concret (visée). Le candidat est prêt à travailler dans le domaine de la mécanique, de la maintenance des espaces verts et dans l'agriculture. Cette annonce permet de saisir le style esthétique du candidat qui définit sa personnalité à travers le choix des formes discursives (ce qu'il met en avant, comment il l'articule).

Après ce premier exemple d'un site général d'annonce, poursuivons notre analyse par l'exploration de sites spécialisés en recrutement.

\section{Exemple 2 : Pôle emploi}

Avant d'examiner les stratégies des candidats, il nous semble nécessaire de comprendre comment l'employeur a accès aux informations mises en ligne. L'étude de la demande commence, pour l'employeur, par une recherche par mots-clés définissant le métier voulu, par exemple : "secrétaire ». Ce type de fonctionnalité implique un premier niveau de tri opéré par l'algorithme du site. Ce dernier impose au candidat de rentrer dans des cases et, dans le cas d'un candidat ayant exercé plusieurs 
métiers (cf. exemple 1), de choisir un seul métier-mot-clé pour pouvoir être repéré par un employeur potentiel.

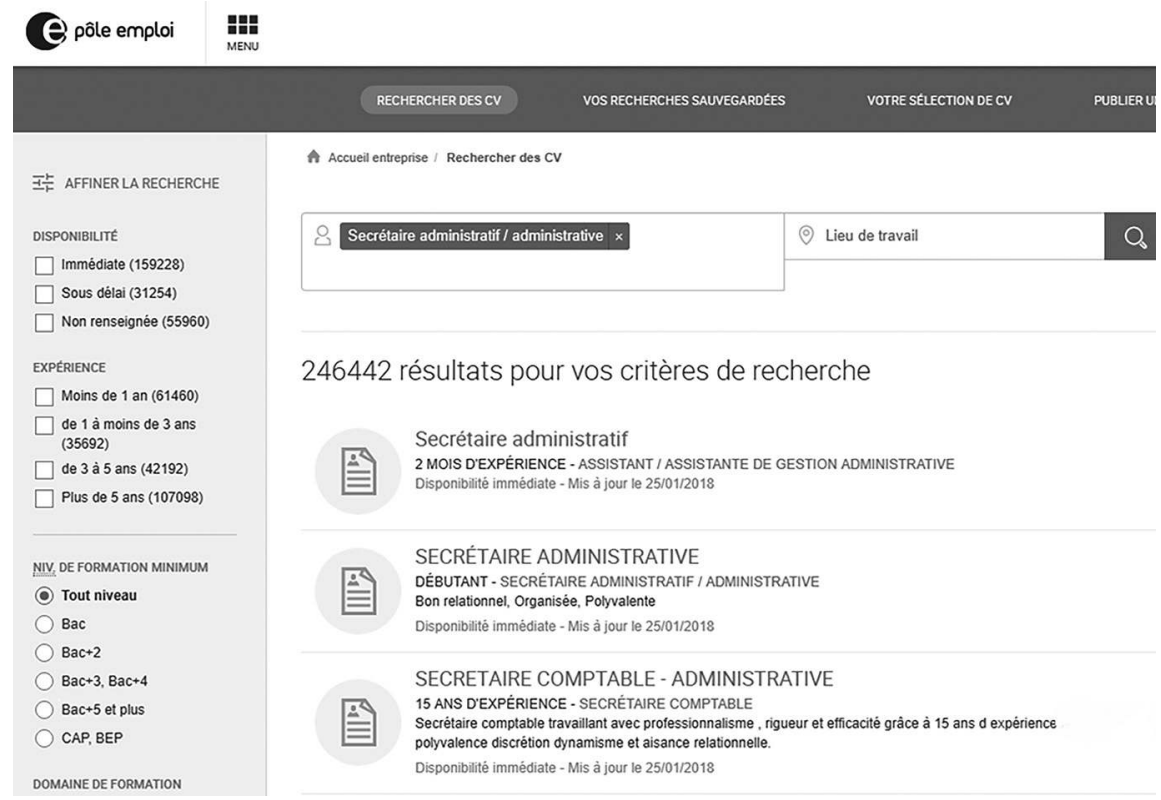

Figure 1 : Base de données de CV pour le poste de « secrétaire » sur le site de Pôle Emploi (source: https://www.pole-emploi.fr/, janv. 2017)

Lécran affiche le nombre de résultats et les critères qui permettent à l'employeur de faire le tri sans avoir lu les annonces proposées. À cette étape les traits personnels ne sont pas accessibles au recruteur. Pour lire le CV l'employeur doit choisir parmi ceux qui lui sont proposés à partir de deux paramètres disponibles : le type de métier (« dans le bâtiment » ou " secrétaire comptable») et l'expérience (en années). Il clique ensuite sur une des candidatures.

En examinant les présentations de candidats, on constate que, contrairement à notre premier exemple, où le récit de vie du candidat était spontané, il est ici intégré à un cadre préétabli et plus strict en terme de forme (modules) et de contenu (compétences et qualités prédéterminées par le métier).Le titre qui définit le métier «secrétaire » est accompagné par un commentaire plus ou moins libre indiquant soit l'expérience (10 ans, grande expérience, etc.), soit les savoir-être : «dynamique, autonome, rigoureuse, discrète ». Ces premières informations sont complétées par : les expériences professionnelles, les formations, les compétences 
avec leur niveau de maitrise (expert, intermédiaire, débutant, avancé), les langues (notions, courant, bon), les permis spécifiques, et enfin, les centres d'intérêt. En voici quelques extraits :

Autonome, disciplinée, organisée et bilingue anglais. Je vous apporte tout mon professionnalisme.

Bonjour, Je suis Saliha! Motivée, dynamique, toujours de bonne humeur et très souriante! De nature enjouée, je suis très impliquée dans les projets que l'on me confie et j'apprécie tisser des relations cordiales avec mes collègues.

De nature dynamique, j'aime les traitements de texte, archives, classement tout ce qui concerne le secrétariat.

J'ai une longue expérience (35 ans au sein du même cabinet médical avec différents employeurs), je suis très patiente et à l'écoute, je suis capable de travailler seule en toute autonomie, ou en équipe, je suis à l'aise dans toutes les tâches de secrétariat.

Ainsi, insérée dans la grille des critères préétablis, faciles à traiter par le robot, l'identité discursive du candidat perd une partie de son caractère personnel en s'ajustant au cadre préétabli. Ici l'ethos montré du candidat apparait seulement dans le commentaire du métier (" dynamique ", " autonome ", etc.). Ce commentaire permet de juger la façon dont les candidats valorisent leur relation à l'autre pour mettre en valeur des compétences propres à un domaine socioprofessionnel particulier. Les termes employés ne permettent toutefois pas de définir le caractère du candidat, ni son projet de vie, mais correspondent plutôt à des valeurs et les qualités génériques jugées importantes pour leur métier. Malgré tout, certains traits personnels du candidat peuvent apparaître dans les commentaires. Dans le deuxième commentaire, le candidat peut être caractérisé comme une personne cordiale en imitant une situation de communication «bonjour » puis en affirmant directement qu'elle apprécie les relations cordiales avec ses collègues. C'est donc bien la construction du discours, plutôt que son contenu, qui permet de qualifier le locuteur.

Pour s'assurer une bonne visibilité et mettre en valeur son profil, le candidat doit ajuster son identité à la manière dont le site trie et classe l'information. C'est pour cela que certains sites (Monster) possèdent des conseillers, qui apprennent au candidat à faciliter la lecture du CV par un moteur de recherche ${ }^{7}$.

7- https://www.monster.fr/conseil-carriere/article/cv-comment-faciliter-sa-lecture-par-unmoteur-de-recherche 
La forme très simple des $\mathrm{CV}$ de Pôle emploi, identique pour tous les candidats (formulaire avec modules), confirme que cette exigence de s'adapter aux contraintes du support efface les traits identitaires personnels propres à chaque candidat qui se manifestent par des choix de style esthétique (formes, couleurs, typographie, images, liens externes).

Nos exemples mettent en évidence, que le candidat doit en premier lieu s'adapter aux contraintes du site (cadres, modules) qui déterminent le degré de liberté dans l'expression de son identité.

\section{Ajustements positionnels par rapport à l'autre : type de l'hétéroaccomo- dation}

\section{Positionnement par rapport au corps de métier}

Les discours étudiés montrent également la tentative du candidat d'appartenir aux identités collectives propres à une profession. Fusiller discute la notion d'ethos professionnel qui selon lui " constitue un dénominateur commun à un ensemble d'individus pratiquant une activité similaire qui se reconnaissent et sont reconnus comme membres d'un groupe professionnel » (Fusilier 2011). Il s'agit donc de formes identitaires réglées déterminant ce qui fait qu'on admire, qu'on estime ou qu'on méprise ses pairs (Zarca 2009:352). Dans le domaine numérique, Domenget constate que les formes identitaires professionnelles renvoient à des visions de soi par les autres mais aussi des autres par soi (Domenget 2015).

Le site LinkedIn, par exemple, devient un outil de ce processus puisque l'identité d'un usager inscrit sur le site est soumise au regard des membres de son réseau, dont le jugement (compétences, «endorsements») construit la réputation aux yeux du recruteur. Plus l'identité du candidat est conforme aux valeurs et aux règles d'un métier, mieux elle sera évaluée par les membres de la communauté.

\section{Positionnement par rapport aux valeurs de l'entreprise}

En général, l'identité de l'entreprise recruteur se manifeste dans la rubrique « offre d'emploi » de son site. Quand le candidat répond à une offre, il tente d'ajuster son image à celle d'entreprise. Dans ce cas-là, le candidat choisi sera celui qui a pu le mieux cerner les attentes, les valeurs et les exigences de l'entreprise. On peut considérer qu'un 
candidat se présentant par candidature spontanée s'affranchirait en partie de la nécessité de se conformer à un profil bien défini. Il aurait alors la possibilité d'intégrer au mieux son projet de vie aux exigences de l'entreprise. En conséquence, sa sélection de contenus représentant son identité serait moins exigeante ; on peut également penser que la compatibilité entre l'employeur et l'employé serait davantage éthique que fonctionnelle (occuper un poste).

\section{Positionnement par rapport à l'observateur-intrus : le cas de Facebook}

L'ethos se construit dans l'interaction sociale définie par Goffman comme «l'influence réciproque que les partenaires exercent sur leurs actions respectives lorsqu'ils sont en présence physique les uns des autres » (Goffman 1992 : 64). Or, sur les plateformes numériques, les partenaires ne sont pas en présence physique mais virtuelle, cela présuppose que les personnes réelles sont derrière les simulacres discursifs. Selon Goffman, le cadre social dans lequel se trouvent les partenaires de l'échange leur impose des rôles, des modèles d'action préétablis à l'avance. Par exemple, les rôles de directeur, de juge, de père de famille, etc. On constate alors que la présentation de soi est tributaire des rôles sociaux et des données situationnelles. Elle est soumise à une régulation socioculturelle. En ce sens, Maingueneau affirme que, pour légitimer son dire, l'énonciateur doit s'inscrire dans une scène d'énonciation. "A l'intérieur de ce genre le locuteur peut choisir plus ou moins librement sa scénographie, à savoir un scénario préétabli qui lui convient et qui lui dicte une certaine posture » (Charaudeau, Maingueneau 2002 : 239). Cette citation montre bien que chaque genre de discours comporte une distribution préalable des rôles et que tout locuteur prend position dans un espace social réglé qui détermine son identité possible.

Ainsi, la scénographie adoptée par les utilisateurs de Facebook ou de LinkedIn ne sera pas la même car les participants ne poursuivent pas les mêmes objectifs et ne disposent pas des mêmes rôles. LinkedIn permet de construire un réseau professionnel, Facebook un réseau d'amis avec un caractère plus privé (ou intime). Néanmoins, nous savons que les recruteurs utilisent Facebook pour compléter l'image qu'ils se font du candidat ${ }^{8}$. Du point de vue de l'analyse du discours, la présence du recruteur n'est pas légitime parce qu'il n'est pas le destinataire direct de l'information

8-https://www.cadremploi.fr/editorial/actualites/actu-emploi/detail/article/recruteursfouineurs-quand-les-profils-facebook-importent-plus-que-les-cv.html 
mais un observateur-intrus. Lénonciation du candidat n'est pas orientée vers ce destinataire. Cette pratique a des conséquences puisque certains sites diffusent les conseils de médiateurs soucieux d'ajuster l'image du candidat, sur Facebook, au rôle du candidat à l'emploi. Ils proposent d'intégrer sur la page du candidat des images avec les collègues, dans le bureau, de parler des valeurs professionnelles, de faire un nettoyage du contenu pour effacer toute information pouvant nuire à sa réputation, de sélectionner les photos pouvant mettre le candidat en valeur, et d'entrer en interaction avec les entreprises qui l'intéressent en mettant un like sur leurs pages etc. ${ }^{9}$. Létude montre qu'il y a un lien direct entre le profil des candidats sur Facebook et le nombre de propositions d'embauche ${ }^{10}$.

Nous venons de décrire ici différents types d'ajustements des profils du candidat qui sont bien des hétéroaccomodations produites en fonction des contraintes imposées aux candidats par les pratiques et les supports.

Examinons désormais comment les styles stratégiques des candidats s'organisent en formes de vie au niveau de la culture.

\section{Reconstruire l'image de l'autre : Identité idem / ipse}

Nous sommes partis de l'hypothèse que le discours numérique porte des traces énonciatives qui permettent de définir l'identité du locuteur, le candidat. Dans ce cas les formes et les figures exprimées dans les textes-énoncés pourront nous permettre de définir le projet de vie ou le style identitaires des différents candidats. Le niveau auquel nous allons travailler est celui de la culture qui correspond, chez Fontanille, au niveau d'observation où « la stratégie de l'usager rejoint d'autres stratégies au sein d'une classe plus générale et plus stable dans le temps, dont le plan de l'expression (le style) renvoie à des contenus axiologiques spécifiques, le tout se donnant de saisir comme une forme de vie» (Fontanille 2008 :33).

Ainsi le modèle proposé par Fontanille pour analyser l'identité narrative doit permettre d'identifier les candidats à l'emploi à des types stratégiques. Dans les paragraphes précédents, nous avons montré que l'expérience personnelle, définissant le style individuel du candidat, se manifeste par rapport à un fond des expériences collectives avec lesquelles elle s'accorde ou entre en résonance. Les styles que nous allons essayer de définir vont correspondre (1) aux régimes d'interaction

9-https://frenchemployerbranding.wordpress.com/2013/02/20/annepestel_ marqueemployeur_45/

10-https://www.lexpress.fr/emploi/gestion-carriere/oui-les-recruteurs-examinent-bienvotre-profil-facebook_1609659.html 
entre l'individuel et le collectif, (2) à la constance du caractère et (3) à la fidélité au projet (visée). Nous étudierons les identités des candidats en examinant leur rapport à soi, à l'autre et au métier. Après avoir analysé environ 100 profils nous avons pu les classer en trois catégories. Afin de conserver l'anonymat des profils étudiés, nous leur avons arbitrairement attribué de faux prénoms.

La première position correspond à la « constance ». Elle affiche une domination du Soi-idem, autrement dit, les candidats répètent les mêmes traits tout au long de leur vie. Ils exercent donc généralement le même métier, conforme à leur formation initiale, toute leur vie; leur expérience du même domaine s'enrichit avec le temps ; ils sont plutôt sédentaires (même lieu). Leur parcours montre clairement une répétition des rôles et l'absence de volonté de changement. C'est la raison pour laquelle ces candidats valorisent l'étendue de leur expérience propre à un domaine (ou métier).

Candidats témoins : Nicolas : « Maitrise Word, Excel, Power point, Photophiltre, Logiciel Sage comptabilité Souriante, Dynamique, Qualité d'écoute, Relationnel, Esprit d'équipe, sens de la discrétion, Rigueur, organisation ${ }^{11}$ » ou Audrey : "Plus de 30 années d'expérience! Rigueur, autonomie, conscience professionnelle, esprit d'équipe ».

La position contraire correspond à l'« audace ». Elle se définit par une domination du Soi-ipse. Les candidats de ce type se caractérisent par les termes : changement et innovation. C'est par exemple le cas d'Alexandre qui a obtenu un Master en lettres à l'université à Paris Sorbonne mais a décidé de devenir entrepreneur en créant sa propre boulangerie avec laquelle il a du succès. Il a donc mobilisé son bagage intellectuel dans autre domaine professionnel en s'opposant aux préjugés. Modélisé dans un repère tensif ${ }^{12}$ qui combine un axe de l'étendue en abscisse (quantité, variété des phénomènes) et un axe de l'intensité en ordonnée (force de la visée intentionnelle), cette position se caractérise par une intensité forte et une étendue faible. Une autre candidate affiche sur son profil LinkedIn la volonté de changer : «En recherche de projets fantastiques ou d'un poste incroyable!».

11- Tiré du site Pole emploi : recherche « secrétaire » (consulté le 11/01/2018).

12- Le schéma tensif est un modèle descriptif de l'univers sémantique qui a été introduit par Fontanille et Zilberberg. Ce modèle prend en compte le sujet du discours qui perçoit les phénomènes signifiants comme une présence sensible. Cette présence s'exprime à la fois en termes d'intensité et en termes d'étendue (extensité). (Cf. Signo: http://www.signosemio. com/fontanille/schema-tensif.asp) 
La personne suivante, Céline, occupe la position qu'on nommera «persévérance» manifestée par l'accumulation progressive de compétences dans le même domaine mais aussi des changements de rôles (postes). Son identité se construit par continuité logique, en maintenant la même direction : obtention d'agrégation, puis formation suivie au sein de deux universités, puis activité d'enseignement, de recherche et finalement obtention d'un poste d'enseignant-chercheur. Au niveau de l'expression Céline parle de son expérience en employant la première personne « je », qui montre son implication et son identification au métier. Les descriptions de chaque poste sont assez exhaustives et montrent qu'il s'agit d'une personne rigoureuse et persévérante. Si on analyse le cadre spatial de son parcours de vie, on constate différents lieux de travail (France et étranger), ce qui témoigne de son ouverture et de sa mobilité géographique. Cependant, après avoir voyagé, le candidat revient en France.

Finalement, et de manière un peu plus contrastée, le profil de Céline allie constance et audace. Autrement dit, il conjugue la domination du Soi-idem (répétition) et la domination du Soi-ipse (permanence dans le temps). On observe que le candidat réalise son projet de vie au gré de ses choix, sans s'attacher au lieu, ni au temps mais en suivant le parcours qui lui permet de s'épanouir.

Pour compléter les possibilités, nous devons expliquer la dernière position, représentée par une intensité et étendue faibles, que Fontanille nomme «tendance ». Elle correspond à une sorte de paresse et d'absence de cohérence dans le parcours lorsque le candidat abandonne facilement des projets parce qu'il suit la mode ou ses instincts du moment sans avoir défini sa visée. Cette position est pour notre étude purement théorique dans la mesure où nous n'avons pas trouvé de récits de candidats correspondant. Synthétisons nos observations en représentant ces positions sur un schéma tensif:

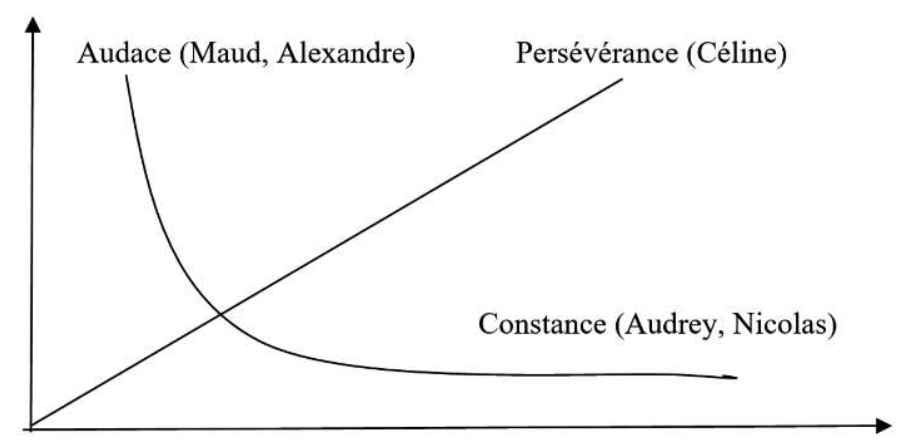

Figure 2 : Schéma tensif des identités numériques (Source : auteurs) 


\section{Conclusion}

Dans cet article, nous avons employé la sémiotique des pratiques pour observer comment les candidats à l'emploi construisent leur identité sur les plateformes numériques. À la suite des recherches en sciences de l'information et de la communication, qui ont décrit l'identité des usagers sur internet et les réseaux sociaux en considérant ses « traces » de navigation comme un discours (Maingueneau, Charaudeau, Domenget), la sémiotique des pratiques considère le media-objet comme un support composite (signe, texte) qui articule les productions textuelles avec des pratiques sociales. L'identité numérique émerge alors comme une identité fluctuante, un ensemble de traits qui s'inscrivent sur le support numérique (pages web, clics). Elle s'adapte au gré de l'expérience de l'usager. Les formes et contenus choisis pour construire son identité deviennent des éléments particulièrement pertinents car, inscrits dans la pratique du recrutement, ils manifestent un style stratégique renseignant à la fois sur la personnalité du candidat et sur ses modes d'ajustement aux attentes de l'entreprise.

Lobservation d'un échantillon de profils sur les plateformes professionnelles (LinkedIn, Monster, Pôle-emploi) a permis d'identifier des types d'ajustements révélant la spécificité des représentations discursives des candidats en ligne. Contrairement à la présence physique, les identités numériques sont des simulacres soumis à des contraintes qui orientent la signification. Notre étude s'est focalisée autant sur les mécanismes de l'ajustement et d'accommodation, qui accompagnent la mise en forme de l'identité numérique, que sur le repérage des 3 types identitaires de candidats à partir desquels nous avons pu définir leur système de valeur et leur façon d'agir.

Létude des styles expérientiels montre la nécessité de saisir les identités numériques au point d'articulation entre leurs dimensions individuelle et collective. La sémiotique des pratiques, qui repose sur l'approche énonciative de l'expérience des usagers, émerge comme un outil adéquat pour saisir la manière dont des individus ou des institutions construisent leur identité par ajustements successifs de leurs discours à l'espace numérique. De la même manière que nous avons dégagé des styles de candidats, nous pourrons poursuivre cette recherche en définissant des styles stratégiques d'entreprises pour préciser la compatibilité des deux ensembles. 


\section{Bibliographie}

AMOSSY Ruth, 2012, L'argumentation dans le discours, Paris, Armand Colin, $346 \mathrm{p}$.

BENVENISTE Émile, 1974, Problèmes de linguistique générale. 2, Paris, Éditions Gallimard, 286 pages.

BEYAERT-GESLIN Anne, 2013, «Quand le candidat devient Président de la République Arguments pour une sémiotique de l'énonciation ", Revue française des sciences de l'information et de la communication, $\mathrm{n}^{\circ} 3$. Disponible sur : https://rfsic.revues.org/540, consulté le 3 janvier 2018.

CHARAUDEAU Patrick, MAINGUENEAU Dominique, 2002, Dictionnaire d'analyse du discours, Paris, Seuil, 661 pages.

DOMENGET Jean-Claude, 2015, « Usages professionnels et figures d'usager des médias socionumériques ", Revue Française des sciences de l'information et de la communication, $\mathrm{n}^{\circ}$ 5. Disponible sur https://journals.openedition.org/rfsic/1325, consulté le 26, 2018.

DONDERO Maria-Giulia, REYES-GARCIA Everardo, 2016, « Les supports des images : de la photographie à l'image numérique, Revue Française des sciences de l'information et de la communication, $\mathrm{n}^{\circ} 9$. Disponible sur http://journals.openedition.org/rfsic/2124, consulté le 5 janvier 2018.

FLOCH Jean-Marie, 1990, Sémiotique, marketing, communication. Sous les signes les stratégies, Paris, Presse Universitaire de France, 233 pages.

FLOCH Jean-Marie, 1995, Identités visuelles, Paris, Presses Universitaires de France, 221 pages.

FONTANILLE Jacques, 2003, Sémiotique du discours, Limoges, Presses Universitaires de Limoges, 303 pages.

FONTANILLE Jacques, 2006, Pratiques sémiotiques : immanence et pertinence, efficience et optimisation, $N A S$ n $^{\circ} 104,105,106$, Limoges, Presses Universitaires de Limoges.

FONTANILLE Jacques, 2008, Pratiques sémiotiques, Paris, Presses Universitaires de France, 303 pages.

FONTANILLE Jacques, 2010, "L'analyse des pratiques : le cours du sens ». Protée, volume 38, numéro 2, automne, 2010, p. 9-19. Disponible sur https://www.erudit.org/fr/revues/pr/2010-v38-n2pr3967/044947ar/resume/

FONTANILLE Jacques, 2011, Corps et sens, Paris, Presses Universitaires de France, 186 pages.

FOURMENTRAUX Jean-Paul (dir.), 2015, Identités numériques. Expression et traçabilité, Paris, CNRS Éditions, 238 pages.

FUSULIER Bernard, 2011, "Le concept d'ethos. De ses usages classiques à un usage renouvelé ", Recherches sociologiques et anthropologiques, n 42-1. Varia. Disponible sur http://journals.openedition.org/rsa/661?lang=en 
GALINON-MELENEC B., MONSEIGNE A, 2011, " La sémiotique des « signes-traces » appliquée au recrutement : Le cas de la recherche du «bon candidat " via les traces numériques ", Communication et organisation, $\mathrm{n}^{\circ} 39$, consulté le 6 janvier 2018.

GOFFMAN Erving, 1959, La mise en scène de la vie quotidienne. 1, La présentation de soi, Paris, Édition de minuit, 1992 [éd. orig : The presentation of self in everyday life].

JEANNERET Yves, 2011, "Complexité de la notion de trace. De la traque au tracé », dans Béatrice Galinon-Mélénec (dir.), L'homme trace. Perspectives anthropologiques des traces contemporaines, Paris, CNRS Éditions, 410 pages.

KERBRAT-ORECCHIONI Catherine, 1980, L'énonciation. De la subjectivité dans le langage, Paris, Armand Colin. 290 pages.

KERBRAT-ORECCHIONI Catherine, 2002, "Rhétorique et interaction ", dans R. Koren et R. Amossy (éds.), Après Perelman : quelles politiques pour les nouvelles rhétoriques? Paris, L'Harmattan, p. 173-196.

MAINGUENEAU Dominique, 2015, « L'ethos discursif et le défi du Web », Itinéraires, $n^{\circ} 3$. Disponible sur https://itineraires.revues.org/3000, consulté le 6 janvier.

PIGNIER Nicole, 2006, " Pour une approche sémio-pragmatique de la communication. Le traitement sur le Web des publicités pour Parfum, Questions de communication ", n ${ }^{\circ} 9$. Disponible sur http://journals.openedition.org/ questionsdecommunication/7945, consulté le 6 janvier.

RICCEUR Paul, 1990, Soi-même comme un autre, Paris, Éditions du Seuil, 424 pages.

ZARCA Bernard, 2009, "L'ethos professionnel des mathématiciens ", Revue française de sociologie, volume 50, $\mathrm{n}^{\circ}$ 2, p. 351-384. Références bibliographiques. 